\title{
Evaluación de la vulnerabilidad sísmica de la tapia en Pasto (Nariño, Colombia). Caso Teatro Imperial - Ingeniería y Patrimonio ${ }^{1}$
}

\section{Evaluation of the seismic vulnerability of the tapia in Pasto (Nariño Colombia) case Imperial Theater-Engineering and Heritage}

\section{Avaliação da vulnerabilidade sísmica da fita em Pasto (Nariño, Colômbia). Caso Teatro Imperial - Ingeniería y Patrimonio}

\author{
W. A. Castillo, G.A. Palma, H. C. Moncayo \\ Recibido: febrero 20 de 2018 - Aceptado: junio 29 de 2018
}

\begin{abstract}
Resumen - En San Juan de Pasto, zona de alta sismicidad, las edificaciones en tierra constituyen un valor de interés cultural de la nación; la mayoría de las edificaciones históricas en Colombia son de este tipo, como el teatro Imperial (1922). Por lo anterior, es un reto para la ingeniería colombiana y para el Ministerio de Cultura, determinar la vulnerabilidad sísmica para regular la protección del patrimonio; sin embargo, la Norma Sismo Resistente del país no reglamenta el análisis y recuperación estructural por falta de investigación. Este artículo ofrece como respuesta, la evaluación estática y dinámica de los materiales a la luz de las estructuras, para determinar la vulnerabilidad sísmica de la tapia. Así, se resuelve
\end{abstract}

${ }^{1}$ Producto derivado del proyecto de investigación "Evaluación de la vulnerabilidad sísmica de la tapia en Pasto (Nariño-Colombia), presentado por el Grupo de Investigación G7, escuela de Posgrado de Ingeniería y Geomática de la Universidad del Valle, como resultado de la comisión de estudios del profesor William Castillo Valencia de la Universidad de Nariño.

W. A. Castillo, Universidad de Nariño, Pasto, Colombia, email:wcastillo2007@gmail.com

G. A. Palma, Universidad del Valle, Cali, Colombia, email: gilberto. areiza@correounivalle.edu.com

H. C. Moncayo, Universidad del Valle, Cali, Colombia, email: ingesuelos1@gmail.com

Como citar este artículo: Castillo, W. A., Palma, G.A. y Moncayo, H. C. Evaluación de la vulnerabilidad sísmica de la tapia en Pasto (Nariño, Colombia), Entre Ciencia e Ingeniería, vol. 12, no. 24, pp. 77-88, juliodiciembre, 2018. DOI: http://dx.doi.org/10.31908/19098367.3816

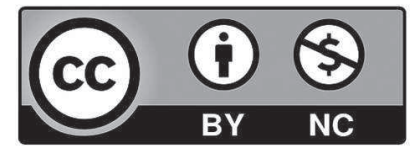

Attribution-NonCommercial 4.0 International (CC BY-NC 4.0) con caracterización físico mecánica mediante la mecánica de la fractura y ensayos de velocidades ultrasónicas, para proyectar métodos de consolidación con base en un análisis no lineal y obtener las curvas con capacidad de desempeño, la relación media de daño y propiciar métodos de consolidación con criterios de respuesta sísmica, índice de sobre esfuerzos, y costos de intervención a que están condicionados.

Palabras clave - amenaza, patrimonio, rehabilitación, sismicidad, tapia, vulnerabilidad.

Abstract - In San Juan de Pasto, an area of high seismicity, the buildings on land constitute a value of the nation's cultural interest; Most of the historical buildings in Colombia are of this type, such as the Imperial Theater (1922). Therefore, it is a challenge for Colombian engineering and for the Ministry of Culture to determine seismic vulnerability to regulate the protection of heritage; however, the Sismo Resistente Norm of Colombia does not regulate structural analysis and recovery due to lack of research. This article offers as response the static and dynamic evaluation of the materials, in light of the structures to determine the seismic vulnerability of the wall, this is resolved with the mechanical physical characterization by the mechanics of the fracture, ultrasonic speed tests, for project consolidation methods, based on a non-linear analysis and obtain the curves with performance capacity, the average damage ratio, and thus promote consolidation methods with seismic response criteria, over-effort index, and intervention costs They are conditioned

Keywords - heritage, seismicity, threat, vulnerability, earth wall, rehabilitation.

Resumo- Em San Juan de Pasto, uma área de alta sismicidade, os edifícios em terra constituem um valor do interesse cultural da nação; A maioria dos edifícios históricos da Colômbia são desse tipo, como o Teatro Imperial (1922). Portanto, é um desafio para a engenharia colombiana e para o Ministério da Cultura determinar a vulnerabilidade 
sísmica para regular a proteção do patrimônio; no entanto, a Norma Sismo Resistente de Colombiana não regulamenta a análise estrutural e a recuperação devido à falta de pesquisa. Este artigo oferece como resposta a avaliação estática e dinâmica dos materiais, à luz das estruturas para determinar a vulnerabilidade sísmica da parede, esta é resolvida com a caracterização física mecânica pela mecânica da fratura, testes de velocidade ultra-sônica, por métodos de consolidação de projeto, baseados em uma análise não linear e obter as curvas com capacidade de desempenho, a taxa média de danos e, assim, promover métodos de consolidação com critérios de resposta sísmica, índice de esforço excessivo e custos de intervenção Eles são condicionados.

Palavras chave - herança, sismicidade, ameaça, vulnerabilidade, muro de terra reabilitação.

\section{INTRODUCCIÓN}

$\mathrm{L}$ A tapia es un sistema constructivo ancestral empleado desde la época prehispánica y modificado durante la conquista española, que se fabrica con una mezcla de materiales: grava, arcilla, arena, limo, agua y materia orgánica, como fibras naturales o animales, para absorber los esfuerzos reólicos, así como el estiércol de vaca y caballo. Este último presenta una composición diferente, ya que el primero es procesado por cuatro cavidades de un estómago y el segundo por uno. Otras sustancias, como la sangre de res, se emplearon como material orgánico plastificante; y la clara de huevo, la melaza y la miel como materiales aglutinantes que, una vez reaccionan químicamente, cumplen la función de endurecer (Fig. 1).

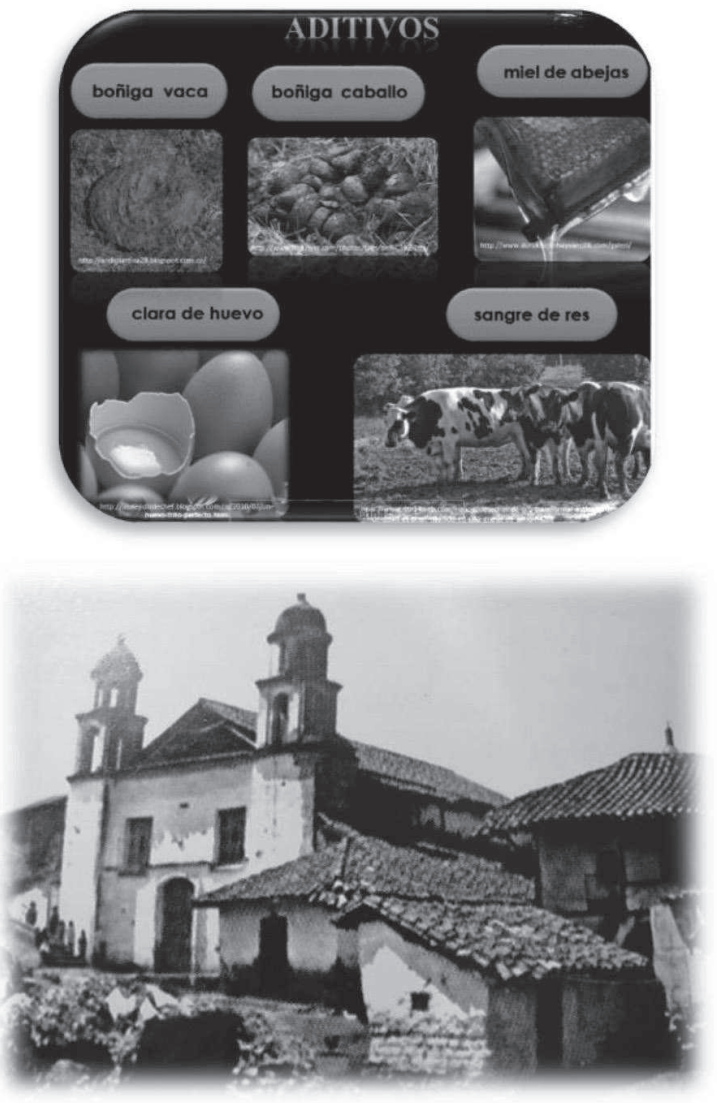

Fig. 1. Elementos aditivos para la fabricación en tapia (izquierda); Casona de Taminango (derecha) (Areiza, Castillo y Coral, 2017).
El $80 \%$ de las edificaciones patrimoniales en Colombia está fabricado en tierra [1], y de dicho porcentaje Pasto posee construcciones históricas que datan del año 1667 [2]. Este tipo de estructuras ha sido sometido a grandes efectos naturales, como el sismo de 1906, caracterizado como uno de los tres terremotos que mayor energía ha liberado en el mundo [3].

Con relación al estado del arte, la conservación y protección de las edificaciones históricas en Pasto logró que estas fueran declaradas como patrimonio nacional. Entre ellas se encuentra el Museo Taminango (1632), Conjunto La Milagrosa (1886), Teatro Imperial (1922) (Fig. 2), y bienes de interés cultural del municipio, como Templo de San Juan (1669) [2], que se construyeron en tierra total o parcial, y mampostería con argamasa de cal y madera. Por su parte, edificaciones en tierra con valor histórico de la ciudad como las casonas Joaquín María Pérez, del consejo municipal y el centro histórico del Colorado, se conservan parcialmente; sin embargo, varias de tales construcciones han sido demolidas por acción antrópica, abandono premeditado de los propietarios, falta de normatización de tipo estructural, inclemencia del clima y efectos naturales. Estos fenómenos amenazan la integridad humana, pues hacen desaparecer bienes con historia y memoria.
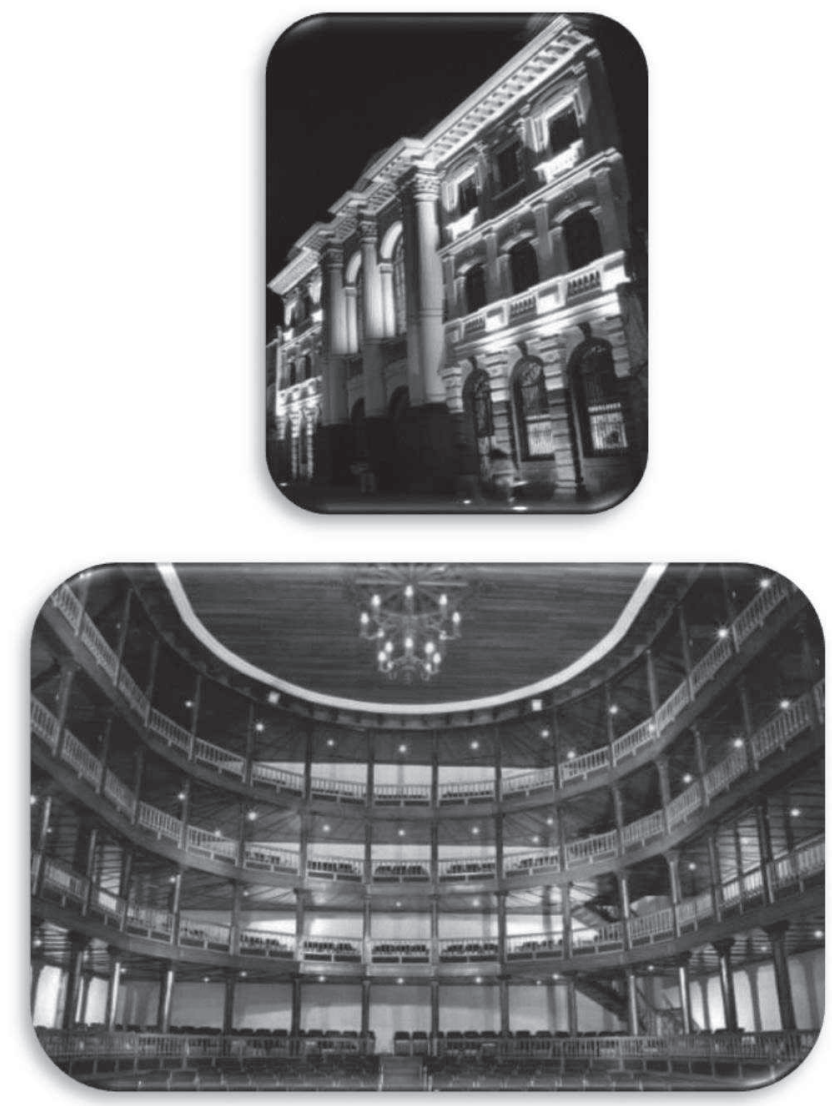

Fig. 2. Fachada y Panorámica interna del sistema estructural del Teatro Imperial - Pasto (Pasuy y Castro, 2003)

Por tanto, para salvaguardar el patrimonio en Pasto, es importante determinar la vulnerabilidad sísmica de las edificaciones en tapia declaradas como bien de interés 
cultural de la nación. En este artículo se toma el caso de estudio del Teatro Imperial, construido en una zona sísmica alta, que ha sido escenario para la presentación de artistas nacionales e internacionales, de reinados, encuentro de toros contra tigres, reuniones políticas (en él se presentó Jorge Eliecer Gaitán), etc.

El bien inmueble era de propiedad en aquel entonces, de don Rafael Villota Chaves (1876-1938) (Fig. 3), y estaba construido principalmente en tapia, mampostería con liga de argamasa de cal (fachada) y madera. Su diseño arquitectónico es obra del ingeniero santandereano Belisario Ruiz Wilches (1887-1958), fundador del Instituto Geográfico Agustín Codazzi en (1935), y del Instituto de Geofísica de la Universidad Nacional de Colombia, y responde a una edificación en forma de "U", tipo teatro carralero español, de cinco pisos, compuesto por una platea y cuatro niveles de palcos. Fue construido por Juan Carlos Molina, [4]: " $\mathrm{El}$ Teatro Imperial fue durante los primeros años del siglo $X X$, la sala exclusiva para proyectar las primeras peliculas del cine mudo... En ese tiempo, el teatro carecía de techo y el público rogaba al cielo para que no lloviera”. El teatro se construyó diez años después (Fig. 4).

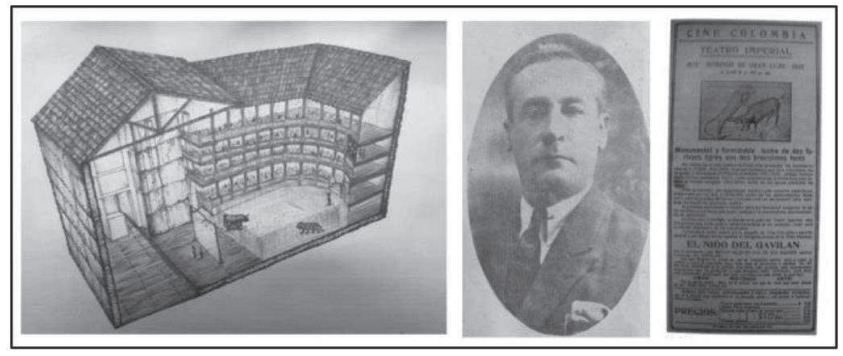

Fig. 3. Representación Ilustrativa Teatro Imperial (Castillo, Burbano y Quiroz, 2017) y volante publicitario original del teatro (Muñoz, 1933)

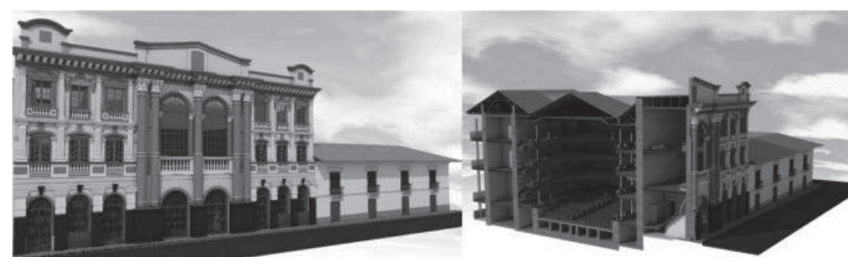

Fig. 4. Teatro Imperial, maqueta virtual, sistema estructural en tapia, madera y mampostería con argamasa de cal.

Con base en la configuración arquitectónica del teatro, en el año 1922 el escenario respondía a la visión espacial de la época; sin embargo, a la luz de la tecnología de la arquitectura teatral, la Universidad de Nariño contrató los servicios del Arquitecto Álvaro Tobón Hincapié, quien modificó la caja del escenario en pro de la disposición funcional, ampliando la profundidad a más del doble e incrementando la altura a 15 metros (punto más alto). La intervención se realizó con estructura independiente en concreto y mampostería dilatada de la estructura principal en tierra; además, se utilizaron algunos perfiles en acero como elementos reversibles.

A la fecha, el teatro presenta serias fracturas (Fig. 5) causadas por las demoliciones periféricas, con probabilidad sísmica de desplome de algunos muros: "El colapso de un muro solamente se producirá cuando el arrostramiento es insuficiente para modificar y atenuar la respuesta dinámica, degradando el material por fisuración, lo que resta posibilidad de estabilidad y se produce el colapso" [5].

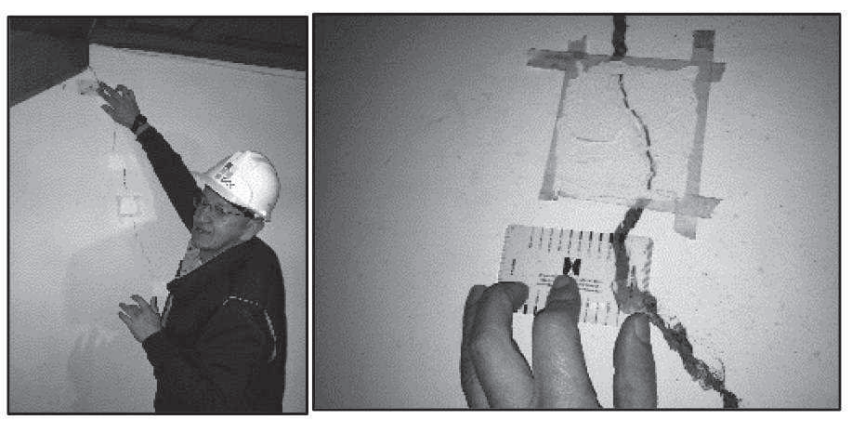

Fig. 5. Verificación de testigos.

De otro lado, el Ministerio de Cultura de Colombia regula la protección de este tipo de edificaciones patrimoniales, pero la Norma de Diseño y Construcción Sismo Resistente [6] no reglamenta la evaluación y recuperación estructural de los mismos.

Con base en un estudio de vulnerabilidad sísmica, la NSR-10 permite que la edificación histórica tenga un menor nivel de seguridad sísmica, respaldado por un memorial cruzado entre el ingeniero diseñador y el propietario. Ante la ausencia de documentación sobre el tema, se hace referencia a las cartas internacionales de restauración, tales como Venecia, Washington y Quito; y a los lineamientos del Comité Científico Internacional para el análisis y restauración de estructuras históricas, ISCARSAH de ICOMOS, en el cual se argumenta que todo proyecto de restauración y conservación requiere de una total comprensión del comportamiento estructural y de las características de los materiales. También, es esencial tener información sobre la estructura en su estado original y en los estados anteriores a la intervención, así como sobre las técnicas que se utilizaron para su construcción, las alteraciones y sus efectos, los fenómenos que la han afectado y, finalmente, su estado actual [7].

Es importante, entonces, conocer el comportamiento estructural de este tipo de edificaciones en tierra para ofrecer herramientas de evaluación de vulnerabilidad estructural, desarrollar experimentalmente bases teóricas, producir documentos de apoyo y establecer metodologías de análisis.

Por lo anterior, en el análisis de los macro elementos del teatro Imperial (Fig. 6), este ha sido intervenido con diferentes materiales propios de cada época, como mampostería, concreto (como la ampliación de la caja del escenario) y concreto en la zona posterior del antiguo hotel; la cubierta se reemplazó en un 95\% y la teja de barro por tejas en asbesto cemento. El concepto de los macro elementos permite evaluar el trabajo: "Las estructuras históricas tienden a comportarse como unidades repetitivas con un comportamiento independiente, concepto mundialmente aceptado que da origen a la definición de macro elementos" [8]. 


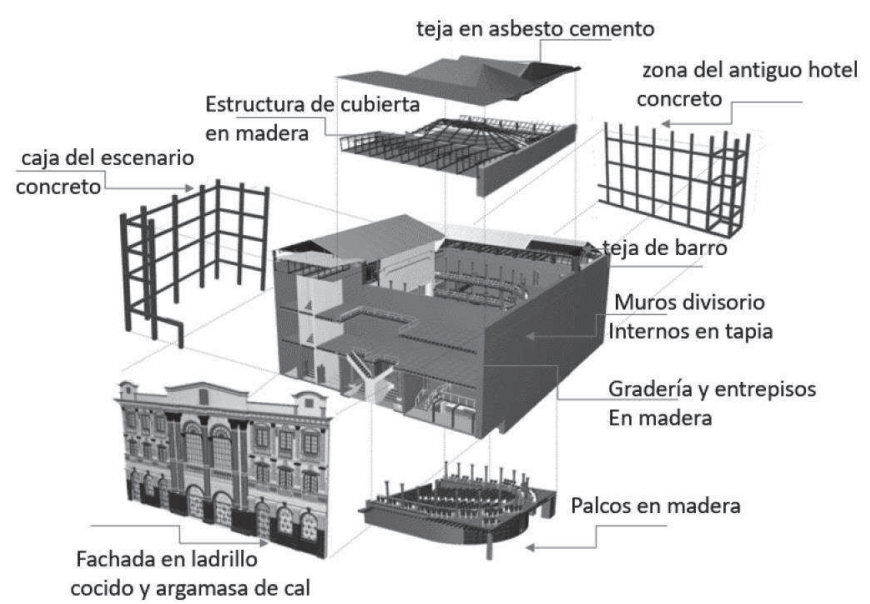

Fig. 6. Macro elementos del teatro Imperial.

\section{METODOLOGÍA}

\section{A Evaluación físico mecánica de la tapia (estática y} dinámica)

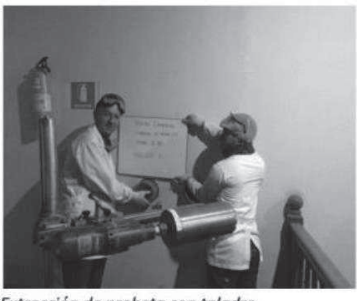

Extracción de probeta con taladro

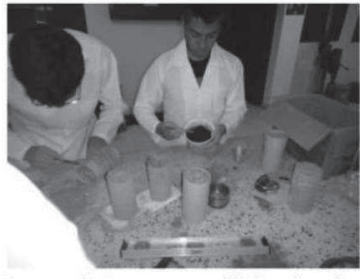

Preparación de muestras perfilado y alineado

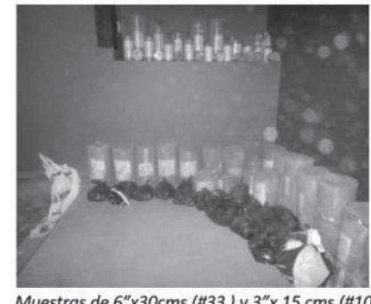

Muestras de 6"x30cms (\#33) y $3^{\prime \prime} \times 15 \mathrm{cms}(\# 10)$

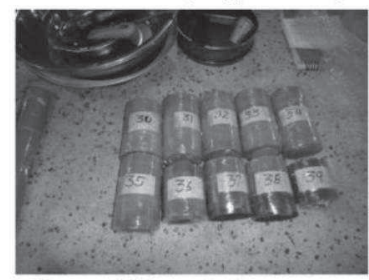

Probetas perfiladas y capinadas para ensayo
Fig.7. Muestreo de probetas en tapia caso teatro Imperial

La tapia se considera como un suelo consolidado (Fig. 7), la cual se somete a ensayos estandarizados o, en caso de no existir, se hace una adaptación de las normas existentes; así, se definen las convencionales como el tipo de suelo, compresión inconfinada, relación de Poisson.

\section{B. Propiedades dinámicas de la tapia}

La dinámica de suelos "es un área que tiene una relevancia importante.... en regiones vulnerables a fenómenos sísmicos" [9]. Es determinante para relacionar los modelos dinámicos con el análisis no lineal del diseño estructural: "cuando una estructura de suelo se ve afectada por cargas cíclicas, se presentan pérdida de su resistencia, cambios de volumen, inestabilidad" [10].

\section{Velocidades ultrasónicas}

"El análisis de ondas del espécimen de muestreo es herramienta fundamental para la aplicación en estudios de riesgo sísmico" [11]. El análisis de la velocidad de onda (P o S: compresión y cortante) permite obtener los parámetros dinámicos, como el módulo dinámico de elasticidad (E), módulo dinámico de esfuerzo cortante $(\mathrm{G})$, coeficiente de Poisson dinámico $(v)$, los parámetros de Lamé $(\lambda)$ y la Constante de Bulk (K).

D. Resistencia a la flexión de la tapia, método de la viga simple cargada en el punto central

Según el Grupo Geotécnica de la Universidad de Cantabria [12]: "La resistencia a tracción de los suelos es prácticamente nula, solamente en los casos especiales de suelos cementados (caso de transición hacia el comportamiento de las rocas)". Para evaluar la flexión de la tapia, se considera como un suelo cementado (Fig. 8) con propiedades especiales, siguiendo los protocolos de flexión.

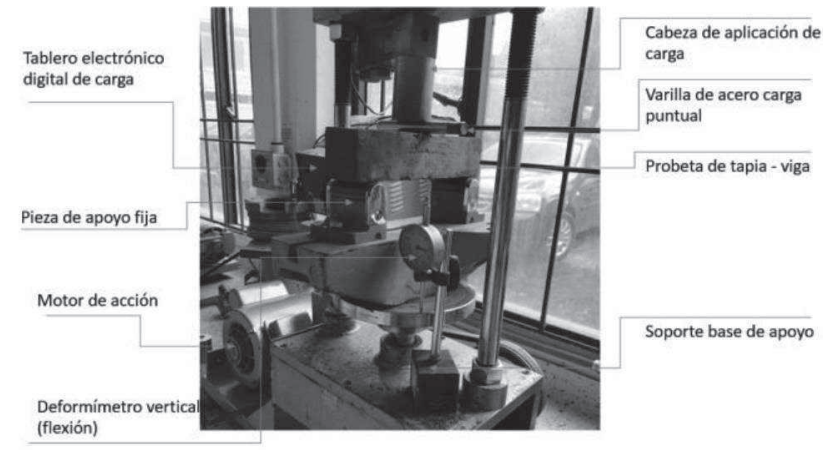

Fig.8. Equipo de ensayo para la probeta de la viga de tapia.

\section{E. Modelo físico mecánico del comportamiento de los muros mixtos sin refuerzo}

En este trabajo se adopta la metodología de la evaluación de las estructuras de mampuestos sin refuerzo [13]. Para el análisis, se evalúa el estado de falla en relación con la deflexión (Fig. 9), así:

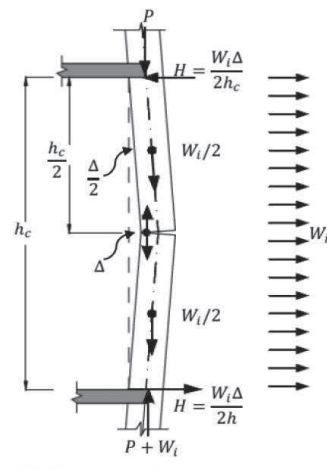

(a) Fuerzas en el muro cargado, incluyendo reacciones laterales

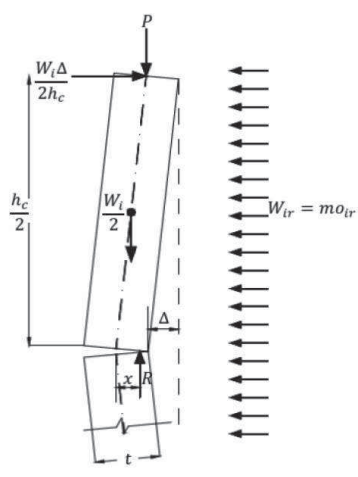
muro cargado (b) Momento de equilibrio para

Fig. 9. Cargas de inercia de respuesta fuera del plano del muro tapial [13].
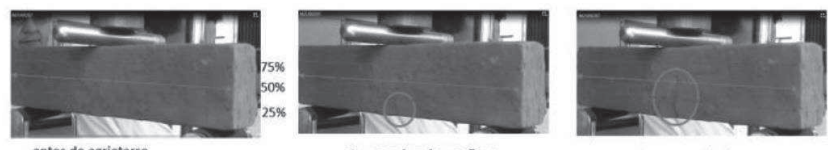

antes de agrietarse

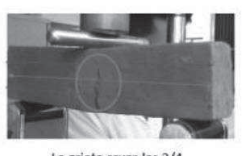

La grieta cruza las $3 / 4$ ta grieta cruza el eje neutro

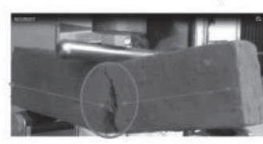

Colapso total

Fig. 10. Mecánica de la fractura - plano de falla-Teatro Imperial. 
Caso 1. El caso antes de agrietarse (Fig. 11-a): la respuesta del esfuerzo por compresión es lineal y se presenta máximo en la sección central del muro.

Caso 2. El semi agrietado (Fig. 11-b): evalúa la distribución de tensiones cuando la grieta se ha propagado al centroide del muro.

Caso 3. El 75\% de la grieta (Fig. 11-c): los cálculos se desarrollan cuando la grieta se ha propagado hasta los tres cuartos de la profundidad de la sección.

Caso 4. Plantea las condiciones últimas hasta el colapso final (Fig. 11-d):

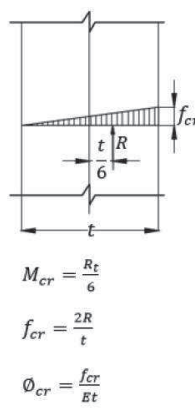

(a) Fisura leve

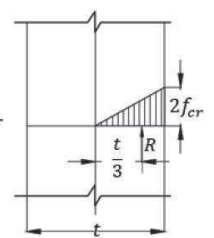

$M=\frac{R_{t}}{3}=2 M_{c r}$

$f_{c}=\frac{2 R}{t / 2}=2 f_{c r}$

$\emptyset=\frac{2 f_{c r}}{E t / 2}=4 \emptyset_{c r}$

(b) Medio agrietado
Momentos y curvatura en el muro en función de la mecánica de la fractura

Fig. 11. Diagrama de esfuerzos momentos y curvatura por estados de la mecánica de fractura [13].

\section{F. Método de los elementos finitos}

Es un método numérico que resuelve ecuaciones diferenciales, relacionando los modelos de cuerpo con geometría sometida a acciones físicas. Esto a partir de una división de dominio en elementos finitos que genera una malla de elementos cada vez más pequeños para aproximarse a la realidad, con procesos experimentales que involucren la estática y la dinámica, podrían aproximar a la respuesta del sistema estructural en tierra.

\section{G. Resultados}

\section{1) Clasificación del suelo de tapia}

La tapia estructura portante del Teatro Imperial, según la clasificación basada en la AASHTO, se determinó como un suelo de características limosas con una clasificación general de tipo A-4. De acuerdo con la clasificación basada en el S.U.C.S. (Sistema Unificado de Clasificación de Suelos), se calificó como un suelo de partículas finas, con símbolo de grupo ML, es decir, un limo de baja plasticidad. A partir de los límites de Atterberg se obtiene el grado de consistencia de cada espécimen analizado; en promedio, media dura, sólida.

\section{2) Propiedades estáticas de la tapia}

Se evalúa la resistencia a la compresión no confinada, la relación de Poisson, el módulo de esfuerzo cortante (Fig.
12), en función del módulo de elasticidad y la relación de Poisson resumidos a continuación (Tabla II).

TABLA I

Límites de consistencia del suelo que constituye la tapia.

\begin{tabular}{lllllll}
\hline \multicolumn{7}{c}{ Límites de consistencia } \\
\hline Material & Limite & Limite & Indice de & Comtenido & Grado de & Grado \\
& Liquido & Plástico & Plasticidad & de & consistencia & de \\
& LL (\%) & LP (\%) & IP (\%) & Humedad & $\mathrm{Kw}$ & consist \\
& & & Wn $(\%)$ & & encia
\end{tabular}

\begin{tabular}{lllllll}
\hline $\begin{array}{l}\text { Promedi } \\
\text { o }\end{array}$ & 42.83 & 30.18 & 12.66 & 18.38 & 2.05 & $\begin{array}{l}\text { Media Dura } \\
\text { Sólida }\end{array}$
\end{tabular}

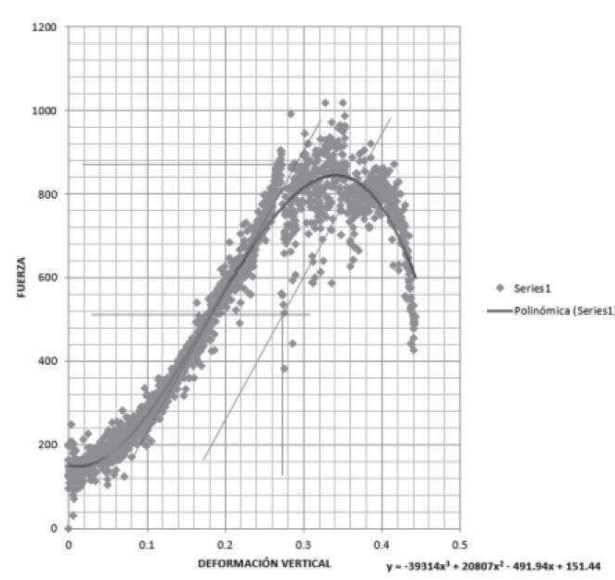

Pistón de carga Tablero de control

Probeta fallada a compresión

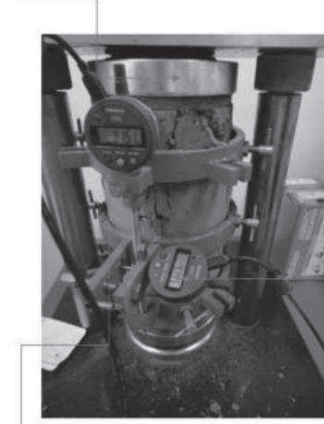
(diámetro $15.24 \mathrm{cmx}$ altura $30.48 \mathrm{~cm}$ )

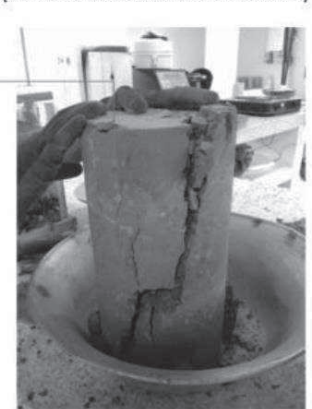

Deformímetro vertical (compresómetro)

Deformimetro horizontal (expansómetro)

Fig. 12. Ensayo de compresión simple curva de fuerza Vs. deformación y la evaluación del módulo tangente. Equipo de ensayo de la relación de Poisson compresión y expansión U. de Nariño.

TABLA II

PARÁMETROS FísICO MECÁNICOS - ESTÁTICOS DE LA TAPIA.

Resistencia Máxima a Compresión, Modulo de Young, Relación de Poisson, Módulo de esfuerzo cortante

\begin{tabular}{|c|c|c|c|c|}
\hline Probeta & $\begin{array}{c}\text { Resistencia } \\
(t / m 2)\end{array}$ & $E(t / m 2)$ & $\mu$ & $G(t / m 2)$ \\
\hline Promedio & 58.92 & 7247.34 & 0.33 & 2766.50 \\
\hline
\end{tabular}

H. Propiedades dinámicas de la tapia

1) Velocidades ultrasónicas

A partir de este ensayo (Fig. 13) se obtiene: el módulo dinámico de elasticidad (E), módulo dinámico de esfuerzo 
cortante $(\mathrm{G})$, coeficiente de Poisson dinámico (v) y los parámetros de Lamé $(\lambda)$ Constante de Bulk $(\mathrm{K})$, como se relacionan a continuación (Tabla III):

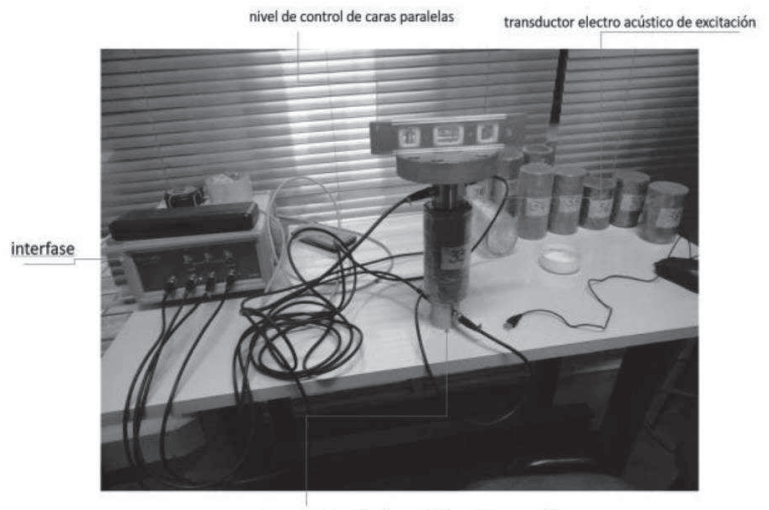

transductor electro acústico de recepción

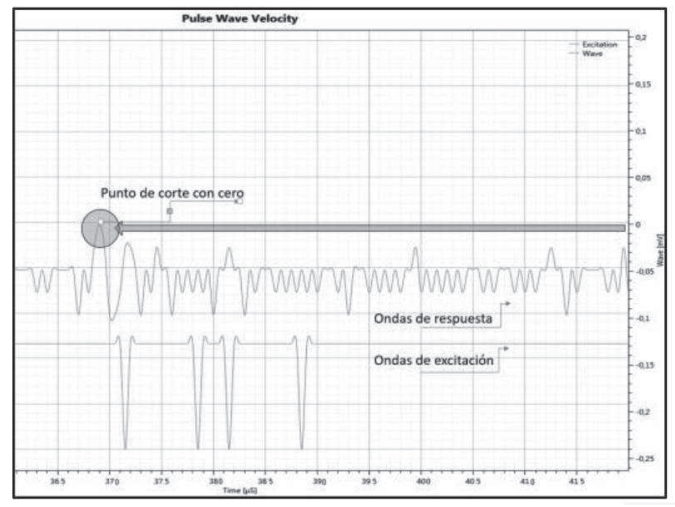

Fig. 13. Equipo de medición de velocidades ultrasónicas Fuente: Esta Investigación William Castillo

Grafica a menor escala para cuantificación de tiempo de recorrido de Ondas $\mathrm{S}$ en espécimen de la muestra: Tapia

TABLA III

PARÁMETRos Físico MECÁNICOS - DINÁMICOS DE LA TAPIA.

\begin{tabular}{|c|c|c|c|c|c|c|c|}
\hline \multicolumn{8}{|c|}{ Parámetros físico mecánicos - dinámicos de la tapia } \\
\hline $\begin{array}{l}\text { Velocidad } \\
\text { de } \\
\text { Compresi } \\
\text { ón }\end{array}$ & $\begin{array}{l}\text { Velocid } \\
\text { ad de } \\
\text { Corte }\end{array}$ & $\begin{array}{l}\text { Módulo } \\
\text { Elasticid } \\
\text { ad } \\
\text { Dinámic } \\
\text { o }\end{array}$ & $\begin{array}{l}\text { Módul } \\
\text { o } \\
\text { Cortan } \\
\text { te G }\end{array}$ & $\begin{array}{l}\text { Relación } \\
\text { de } \\
\text { Poisson( } \\
\mu \text { ) }\end{array}$ & $\begin{array}{l}\text { Constan } \\
\text { te de } \\
\text { Lamé } \\
(\lambda)\end{array}$ & $\begin{array}{l}\text { Módul } \\
\text { o Bulk } \\
\text { (K) }\end{array}$ & Probeta \\
\hline $\mathrm{M} / \mathrm{sg}$. & $\mathrm{M} / \mathrm{sg}$. & Mpa & Mpa & & Mpa & Mpa & Mpa \\
\hline 723.242 & 303.985 & 385.6 & 139.5 & 0.383 & 519.3 & 612.3 & $\begin{array}{l}\text { Promed } \\
\text { io }\end{array}$ \\
\hline
\end{tabular}

El ensayo dinámico se estandariza para medios continuos (en las dos direcciones) de roca o de suelo y, a su vez, es adaptado para la tapia con espesores promedios de $90 \mathrm{cms}$., caracterizado como medio restringido en el sentido del espesor del muro, la cual se expone a cargas dinámicas laterales. Por lo tanto, se recomienda utilizar los parámetros dinámicos como 1/10 del valor estandarizado; por ejemplo, el módulo de elasticidad dinámico: $38.56 \mathrm{Mpa}$ y así cada parámetro de la Tabla III.

2) Resistencia a la flexión de la tapia método de la viga simple cargada en el punto central

Para ello, se calcula el módulo de rotura de la tapia (Fig. 14) con base en la siguiente relación:

$$
R=\frac{3 P l}{2 b d^{\wedge} 2}
$$

\section{Donde:}

$R=$ módulo de rotura, $M P a(p s i)$,

$P=$ máxima carga aplicada indicada por la máquina de ensayo, $N(l b f)$,

$l=$ longitud de la luz, $\mathrm{mm}$ (pulg),

$b=$ ancho promedio del espécimen en el punto de fractura, $\mathrm{mm}$ (pulg), $y$

$d$ = altura promedio del espécimen, en el punto de fractura $\mathrm{mm}$ (pulg).
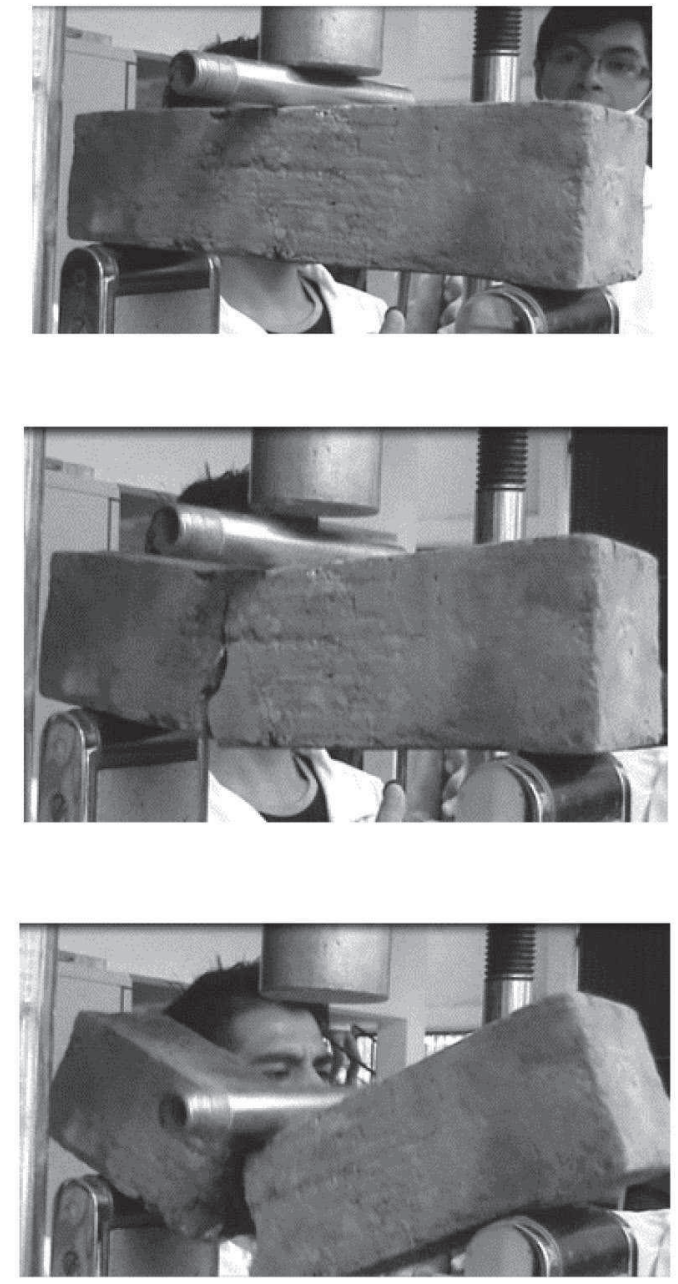

Fig. 14. Secuencia de falla de la probeta de la viga de tapia.

El ensayo de módulo de rotura (Tabla IV) se realiza con probetas obtenidas in situ, en las cuales la fractura que se genera en la viga por las tensiones de la fibra inferior es vertical, buscando la configuración de la matriz de los agregados gruesos. La manifestación de falla en el 17\% es súbita después de alcanzar la máxima resistencia. El 83\% de las probetas presentó un esfuerzo residual después de alcanzar la máxima carga.

El resultado de los ensayos a flexión, para las probetas tipo viga simple (Fig. 15) sin pañete de refuerzo, se describe para cada carga máxima la deformación respectiva, además del cortante, momento flector y el módulo de rotura. 
TABLA IV

ReSULTADO DE LOS ENSAYOS DE FLEXIÓN DE LA TAPIA PROBETA VIGA SI REFUERZO.

\begin{tabular}{|c|c|c|c|c|c|c|c|c|c|c|}
\hline \multicolumn{11}{|c|}{ Viga sin pañete reforzado } \\
\hline \multirow[t]{2}{*}{$\begin{array}{l}\text { Probe } \\
\text { ta }\end{array}$} & \multicolumn{2}{|c|}{$\begin{array}{l}\text { Carga } \\
\text { máxima }\end{array}$} & \multicolumn{2}{|c|}{$\begin{array}{l}\text { Deformació } \\
\text { n alcanzada }\end{array}$} & \multicolumn{2}{|c|}{$\begin{array}{l}\text { Cortante } \\
\text { Máximo }\end{array}$} & \multicolumn{2}{|c|}{$\begin{array}{l}\text { Momento } \\
\text { Máximo }\end{array}$} & \multicolumn{2}{|c|}{$\begin{array}{l}\text { Módulo de } \\
\text { Rotura }\end{array}$} \\
\hline & (N) & $\begin{array}{l}\text { (To } \\
\text { n) }\end{array}$ & $\begin{array}{l}\text { (m } \\
\mathrm{m})\end{array}$ & (m) & $(\mathrm{N})$ & $\begin{array}{l}\text { (To } \\
\text { n) }\end{array}$ & $\begin{array}{l}\text { (N } \\
\mathrm{mm})\end{array}$ & $\begin{array}{l}\text { (Tn- } \\
\text { m) }\end{array}$ & $\begin{array}{l}\mathrm{M} \\
\text { pa }\end{array}$ & $\begin{array}{l}\text { Ton/ } \\
\mathrm{m} 2\end{array}$ \\
\hline Prome & 346. & 0.0 & 1.9 & 0.00 & 173. & 0.0 & 18220 & 0.00 & 0.1 & 13.22 \\
\hline dio & 96 & 35 & 1 & 19 & 48 & 17 & .66 & 18 & 3 & \\
\hline
\end{tabular}

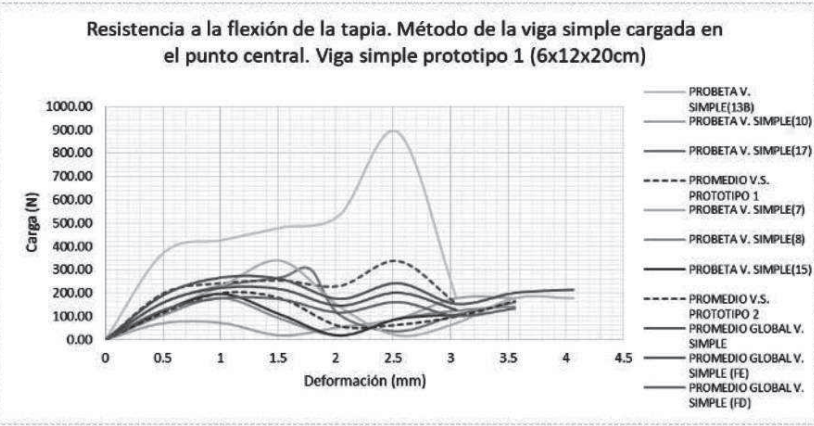

Fig. 15. Curvas esfuerzo deformación de las probetas de las vigas de tapia simple.

Paralelo a la probeta de viga simple se realizó un análisis con los resultados de laboratorio, se aplicó a un espécimen idéntico un pañete de $3 \mathrm{~cm}$ a cada cara con refuerzo en malla de vena (Tabla 6) y otras con malla electro soldada. Para el pañete se utilizó un suelo cemento del $10 \%$ y fibra del $0.5 \%$ en peso; así, la fibra cumple una función importante para absorber esfuerzos reólicos por cambios de temperatura evitando fisuración, ofreciendo un buen aspecto estético. El pañete de suelo cemento que se aplicó por capas compactadas en promedio tiene una resistencia de $1.63 \mathrm{Mpa}$.

En el proceso de aplicación de cargas al material, en el primerinstante, el pañete en suelo cemento reforzado con malla de vena o malla electro soldada (en forma de sándwich) (Fig. 16) asume los esfuerzos en la posición externa de la probeta, aunado a las fibras en polipropileno (Fig. 17), absorbiendo las tensiones iniciales en las diferentes direcciones, cuya composición relaja y disipa hasta transmitir los esfuerzos al núcleo de tapia. Así, con la adición de las fibras al pañete, se consigue disminuir la fisuración ocasionada por retracción plástica. Comparativamente, se realiza una superposición de las curvas de las probetas con pañete reforzado (con malla de vena y una pasta de suelo cemento y malla electro soldada con la misma pasta), para visualizar el estado de respuesta en la curva de esfuerzo de deformación y así se obtiene un promedio del comportamiento (línea punteada en la Fig. 18).

\section{Discusión}

Se obtuvieron los promedios de las curvas como resultado de las probetas simples y reforzadas (A con malla de vena y B con malla electro soldada) (Fig. 22). Las probetas consolidadas con pañete reforzado con malla de vena comparada con aquella reforzada con electro soldada (Fig. 23) son más dúctiles y ofrecen mejores esfuerzos residuales.

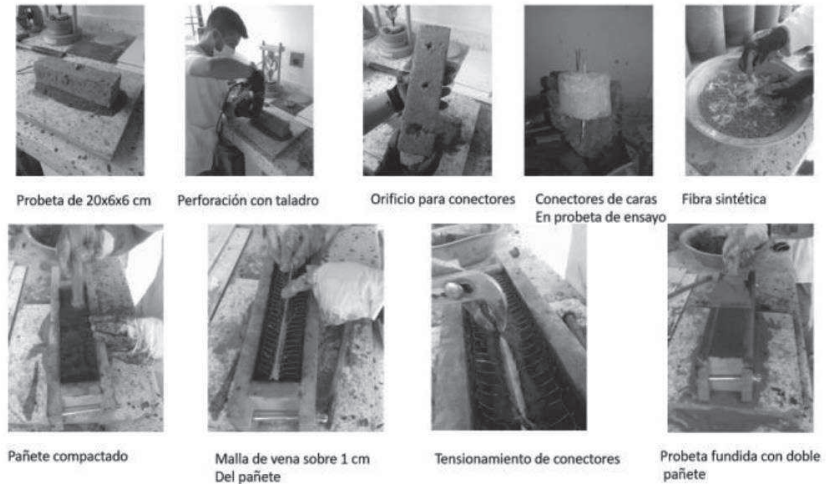

Fig. 16. Proceso de refuerzo con pañete de la probeta de la viga de tapia.
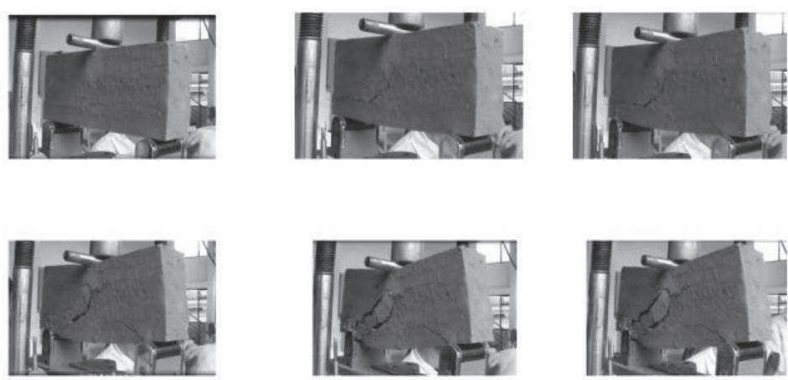

Fig. 17. Secuencia de falla de la probeta de la viga de tapia con pañete reforzado.

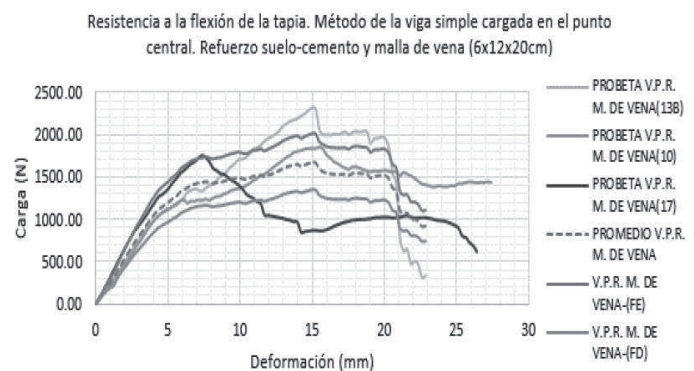

Resistencia a la flexión de la tapia. Método de la viga simple cargada en el punto central. Refuerzo suelo-cemento y malla electrosoldada $(6 \times 12 \times 20 \mathrm{~cm})$

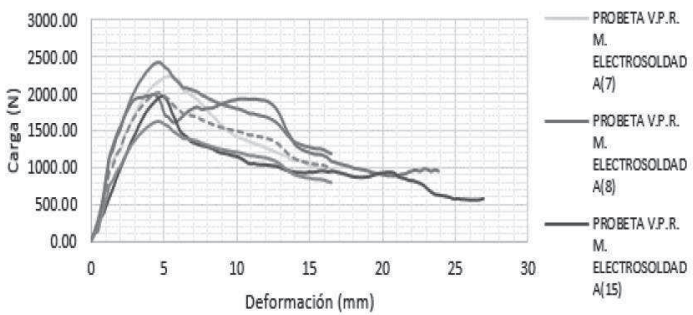

Fig. 18. Superposición de curvas esfuerzo deformación de las probetas de las vigas de tapia con pañete de refuerzo utilizando suelo cemento, malla de vena y malla electro soldada.

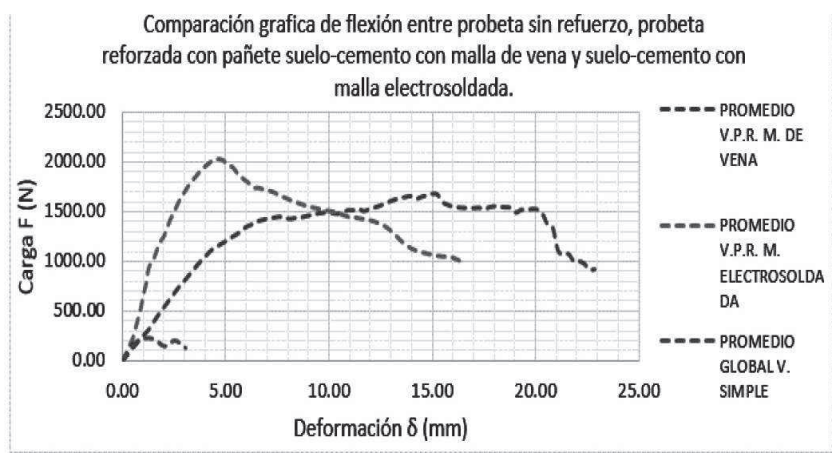

Fig. 19. Curvas esfuerzo deformación de la probeta de la viga de tapia simple y con pañete reforzada con malla electro soldada. 
Para las probetas con pañete reforzado tipo uno (suelo cemento, malla de vena y fibra de polipropileno) (Tabla 5) y tipo dos (suelo cemento, malla electro soldada y fibra de polipropileno) (Tabla 6) en los ensayos a flexión, se describen la carga máxima, la deformación respectiva, el esfuerzo cortante, momento flector y el módulo de rotura, así:

TABLA V

ENSAYO DE FLEXIÓN (PROBETAS TIPO UNO) CON PAÑETE REFORZADO CON MALLA DE VENA Y FIBRA DE POLIPROPILENO

\begin{tabular}{llllll}
\hline \multicolumn{5}{c}{ Viga con pañete reforzado suelo cemento y malla de vena } \\
Probeta & $\begin{array}{l}\text { Carga } \\
\text { Máxi- } \\
\text { ma }\end{array}$ & $\begin{array}{l}\text { Defor- } \\
\text { mación } \\
\text { Alcanzada } \\
(\mathrm{N})\end{array}$ & $\begin{array}{l}\text { Cor- } \\
\text { tante } \\
\text { Máximo }\end{array}$ & $\begin{array}{l}\text { Momen- } \\
\text { to Máx- } \\
\text { imo }\end{array}$ & $\begin{array}{l}\text { Módu- } \\
\text { lo de } \\
\text { Rotura }\end{array}$ \\
$\begin{array}{llllll}\text { Prome - } \\
\text { dio }\end{array}$ & 1977.98 & 12.62 & $1 \mathrm{~N})$ & $(\mathrm{N} \mathrm{mm})$ & Mpa \\
\hline
\end{tabular}

TABLA VI

ENSAYO DE FLEXIÓN (PROBETAS TIPO DOS) CON PAÑETE REFORZADO CON MALLA ELECTRO SOLDADA Y FIBRA DE POLIPROPILENO.

\begin{tabular}{clllll}
\hline \multicolumn{5}{c}{ Viga con pañete reforzado suelo cemento y malla de vena } \\
Probeta & $\begin{array}{l}\text { Carga } \\
\text { Máxi- } \\
\text { ma }\end{array}$ & $\begin{array}{l}\text { Defor- } \\
\text { mación } \\
\text { Alcanzada } \\
\text { (N) }\end{array}$ & $\begin{array}{l}\text { Cor- } \\
\text { tante } \\
\text { Máximo }\end{array}$ & $\begin{array}{l}\text { Momento } \\
\text { Máximo }\end{array}$ & $\begin{array}{l}\text { Módu- } \\
\text { lo de } \\
\text { Rotura }\end{array}$ \\
$\begin{array}{l}\text { Prome- } \\
\text { dio }\end{array}$ & 2071.39 & 4.83 & (N) & $(\mathrm{N} \mathrm{mm})$ & Mpa \\
\hline
\end{tabular}

De los ensayos realizados se obtiene un módulo de rotura con un promedio de $0.74 \mathrm{Mpa}$ para probetas con pañete reforzado con malla de vena; 1.01 Mpa para aquellas reforzadas con malla electro soldada y $0.14 \mathrm{Mpa}$ para probetas simples sin pañete reforzado.

\section{A. Análisis no lineal en el comportamiento y capacidad} de respuesta los muros: tapia y mampostería

Para ilustrar los muros del teatro Imperial por convención, se denomina con la nomenclatura: paralelos a la fachada "Y" y los perpendiculares a esta "X" (Fig. 20).

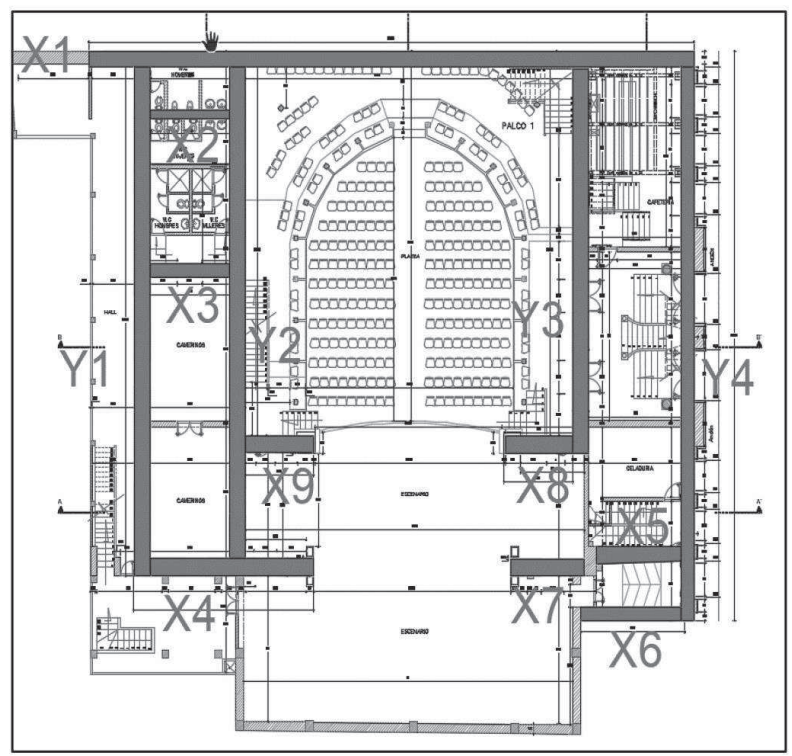

Fig. 20. Convención de nomenclatura para los muros en el plano-Teatro Imperial.
Para interpretar cada una de las gráficas, se seccionan en tres tramos, subdivididas en cuatro zonas: la primera se define como inicialmente agrietado; la segunda indica medio agrietado (la grieta alcanza la mitad del espesor del muro); la tercera indica que la grieta alcanza las tres cuartas partes del espesor del muro; y la cuarta indica el colapso (Fig. 21).

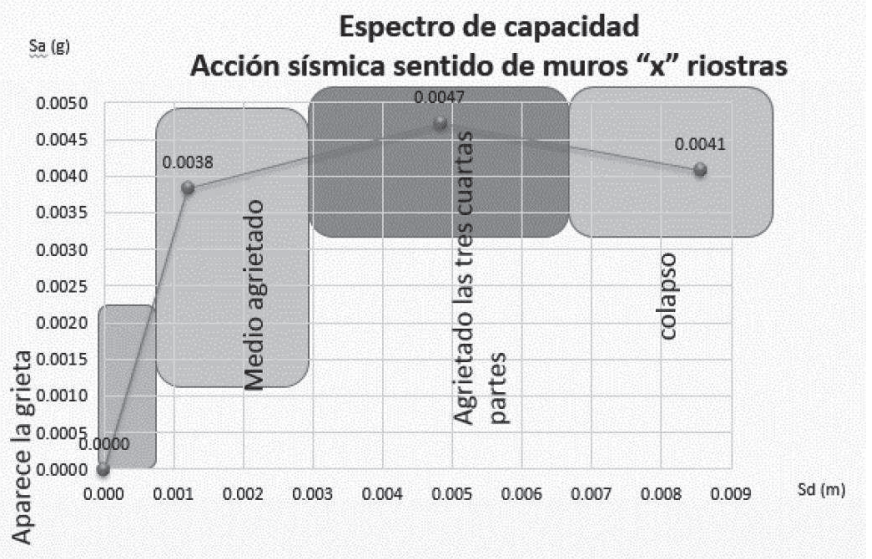

Fig. 21. Convención de nomenclatura para las gráficas de los muros-Teatro Imperial.

B. Curvas de capacidad de muros (acción sísmica sentido $x$ e $y$ )

En la Fig. 22 se muestra un grupo de curvas, "bajas" y "altas"; las primeras, construidas en tierra o tapia, se comportan con grandes deformaciones para un esfuerzo cortante bajo, y aquellos muros de fachada construido en mampostería con unidades en ladrillo cocido y pegados con argamasa de cal representan una curva "alta", con deformaciones moderadas para un esfuerzo cortante relativamente alto.

Curvas de capacidad en el plano Acción sísmica sentido de muros " $y$ " cargueros

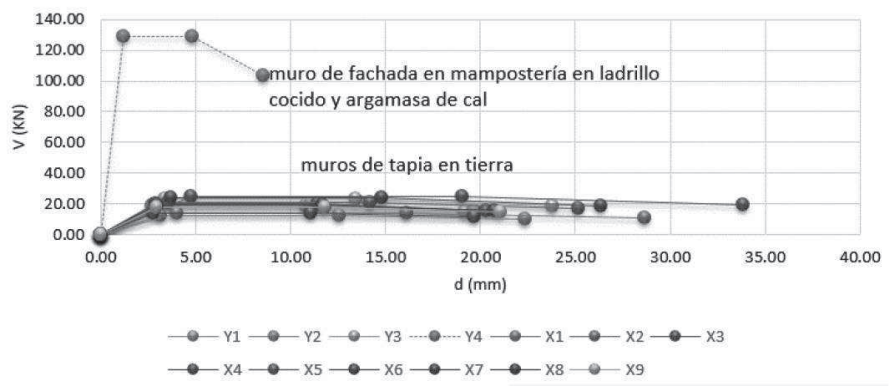

Fig. 22. Curvas de capacidad para muros [14].

\section{Espectro de capacidad}

Para el análisis de los daños de la estructura en tapia, se resuelve la curva del espectro de desplazamiento (Tabla VII) (Fig. 23-b), asociando la aceleración espectral en porcentaje de la gravedad con el desplazamiento; así, para obtener el desempeño sísmico se conjugan el espectro de demanda con el inelástico (en función de la disminución de ductilidad) con base en la suposición de desplazamientos iguales [14]. Para definir los estados del comportamiento de la edificación, 
se asocia a la Relación Media de Daño (RMD), la cual se clasifica como nulo cuando no hay daño, es decir, la $\mathrm{RMD}=0$.

Una relación media de daño es moderado cuando la $\mathrm{RMD}=0.5$, y el estado de falla mediante colapso es $\mathrm{RMD}=1$ (Fig. 23-c y d) [14]. Otra variable que interactúa para definir la curva de vulnerabilidad es el espectro de capacidad en función de la interacción del desplazamiento de los muros, causada por las fuerzas aplicadas a los mismos. Un análisis coherente se debe hacer con tiempos de retorno de 43, 225, 475, 1000 y 2500 años [14]. Luego, sobre el espectro de capacidad de los muros del edificio en análisis se hace una proyección del primer tramo de la línea de periodos cortos y se conjuga con los espectros de 43, 225, 475, 1000 y 2500 (Fig. 23-a), la cual se proyecta hasta la intersección de las abscisas (por cada punto) y se realizan las lecturas en el eje " $\mathrm{X}$ ", para describir los desplazamientos hasta el colapso con base en un tiempo de retorno de la sismicidad.

\section{Curva de vulnerabilidad en función de los espectros de capacidad}

La Relación Media de Daño, RMD, se conjuga con el desplazamiento espectral en función del desempeño; para ello, se calibra la distribución probable con un valor numérico que se ajuste a los datos, según la incertidumbre apoyada en la desviación estándar.

Con base en el desplazamiento proyectado por cada periodo de retorno $(43,225,475,1000,2500$ años), se lo traslada a las curvas de vulnerabilidad, teniendo como parámetro de control la Relación Media de Daño, mediante lo cual se observa que el Teatro Imperial es altamente vulnerable, con base en que acción sísmica sentido de muros "Y" Carguero el desplazamiento $S d$. es de $19.13 \mathrm{~mm}$.

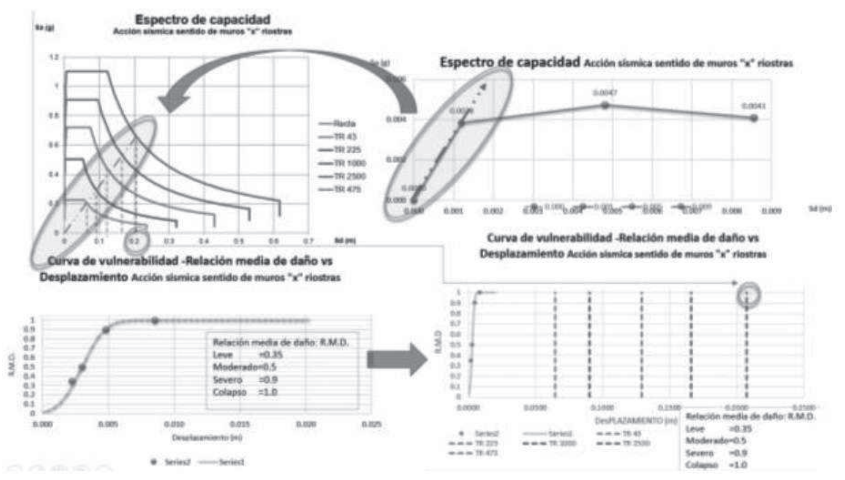

Fig. 23. Curvas para espectro de capacidad - Teatro Imperial [14].
TABLA VII

EVALUACIÓN DEL GRADO DE VULNERABILIDAD CON BASE EN EL ESPECTRO DE DESPLAZAMIENTO SD, EN FUNCIÓN DEL PERIODO DE RETORNO (TR).

\begin{tabular}{|l|c|c|c|c|c|}
\hline \multicolumn{7}{|c|}{ Periodo de retorno (Tr) sentido “y" } \\
\hline TR & TR 43 & TR 225 & TR 475 & TR 1000 & TR 2500 \\
\hline Sd (m) & 0.107 & 0.155 & 0.191 & 0.279 & 0.345 \\
\hline Sd (cm) & 10.729 & 15.468 & 19.127 & 27.893 & 34.527 \\
\hline $\begin{array}{l}\text { Relación media de daño; } \\
\text { RMD }\end{array}$ & 1 & 1 & 1 & 1 & 1 \\
\hline Aparece la fisura & 0.355 & 0.355 & 0.355 & 0.355 & 0.355 \\
\hline Semi fisurado Sd (cm) & 0.920 & 0.920 & 0.920 & 0.920 & 0.920 \\
\hline $\begin{array}{l}\text { La fisura alcanza las Tres } \\
\text { cuartas partes Sd (cm) }\end{array}$ & 1.580 & 1.580 & 1.580 & 1.580 & 1.580 \\
\hline Colapso Sd (cm) & 1.970 & 1.970 & 1.970 & 1.970 & 1.970 \\
\hline
\end{tabular}

Atendiendo a las curvas de capacidad de la estructura, se plantea la hipótesis de falla del Teatro Imperial hasta el colapso, ilustrada en la Fig. 24.

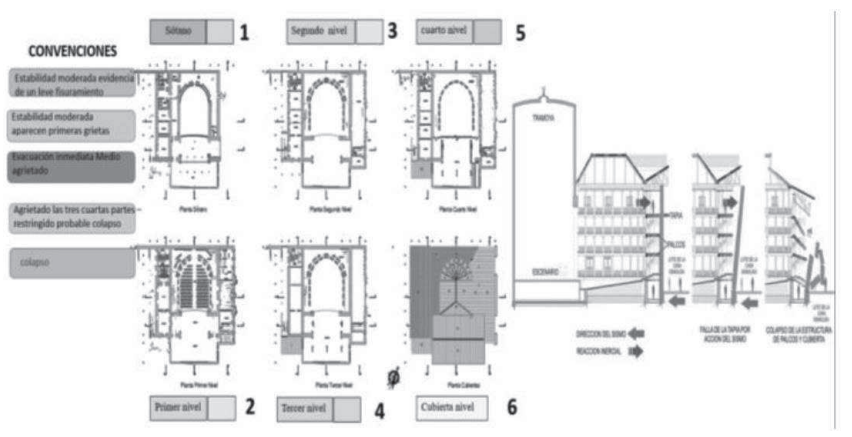

Fig. 24. Hipótesis de falla de la estructura - Teatro Imperial.

El análisis de una estructura en tapia se puede realizar en el rango lineal y no lineal, es decir, que el sistema estructural puede trabajar en el rango elástico e inelástico. Esto significa que se puede llevar a cabo el análisis no lineal cuando no cumple con la ley de Hooke, ya que las cargas causan relajación de la rigidez, y al suspenderlas no se recupera el estado inicial de equilibrio. A la luz de la tecnología aplicada, el análisis no lineal a los muros en tapia implica definir cómo fallará la estructura; por esta razón, se realizó el análisis de la capacidad de los muros en tapia hasta llegar al colapso, entendiendo los tres casos de mecánica de la fractura: aparición de la fisura; la fisura alcanza la mitad del muro; la fisura afecta las tres cuartas partes y el colapso final. El análisis lineal da una idea clara del comportamiento de fuerzas y esfuerzos del teatro para obtener el índice de sobreesfuerzos (Fig. 25) (Tabla VIII).

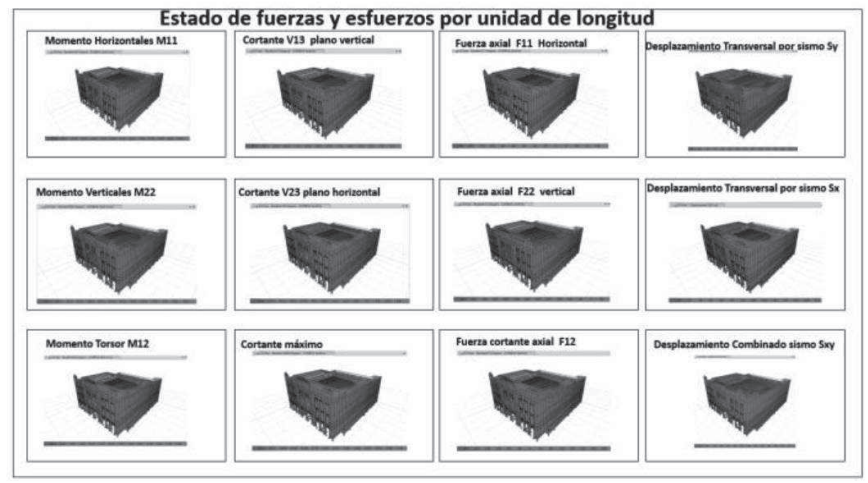

Fig. 25. Esfuerzos en la estructura (FEA) - Teatro Imperial. 
TABLA VIII

EVALUACIÓN DE ESFUERZOS DE LA TAPIA E ÍNDICE DE RESISTENCIA PARA LA TAPIA SIMPLE, PARA LA REFORZADA CON PAÑETE EN MALLA DE VENA Y MALLA ELECTRO SOLDADA.

\begin{tabular}{|c|c|c|c|c|c|c|}
\hline \multicolumn{7}{|c|}{ Evaluación de esfuerzos en la tapia - Chequeo análisis lineal } \\
\hline Esfuerzos & $\underset{(t-m / m)}{\text { M11 }}$ & $\underset{(\mathrm{t}-\mathrm{m} / \mathrm{m})}{\mathbf{M} 22}$ & $V 13(t / m)$ & $V 23(t / m)$ & F11(t) & F22(t) \\
\hline & $\begin{array}{l}\text { Momento } \\
\text { directo } \\
\text { por } \\
\text { unidad de } \\
\text { longitud } \\
\text { horizontal }\end{array}$ & $\begin{array}{l}\text { Momento } \\
\text { directo } \\
\text { por } \\
\text { unidad } \\
\text { de } \\
\text { longitud } \\
\text { vertical }\end{array}$ & $\begin{array}{l}\text { Cortante } \\
\text { fuera del } \\
\text { plano por } \\
\text { unidad de } \\
\text { longitud } \\
\text { horizontal }\end{array}$ & $\begin{array}{l}\text { Cortante } \\
\text { fuera del } \\
\text { plano } \\
\text { por } \\
\text { unidad } \\
\text { de } \\
\text { longitud } \\
\text { vertical }\end{array}$ & $\begin{array}{l}\text { Fuerza } \\
\text { directa } \\
\text { por } \\
\text { unidad } \\
\text { de } \\
\text { longitud }\end{array}$ & $\begin{array}{l}\text { Fuerza } \\
\text { directa } \\
\text { por } \\
\text { unidad } \\
\text { de } \\
\text { longitud }\end{array}$ \\
\hline Actuante & 5 & 4.2 & 7 & 7 & 38 & 53 \\
\hline $\begin{array}{l}\text { Permisible } \\
\text { Simple Sin } \\
\text { Refuerzo }\end{array}$ & 3 & 3 & 6 & 6 & 59 & 59 \\
\hline $\begin{array}{l}\text { Índice De } \\
\text { Sobre } \\
\text { Esfuerzos } \\
\text {-Tapia sin } \\
\text { pañete de } \\
\text { refuerzo }\end{array}$ & 1.67 & 1.4 & 1.17 & 1.17 & 0.64 & 0.9 \\
\hline $\begin{array}{l}\text { Permisible } \\
\text { Pañete } \\
\text { reforzado con } \\
\text { Malla de Vena }\end{array}$ & 4.26 & 4.26 & 8.52 & 8.52 & 59 & 59 \\
\hline $\begin{array}{l}\text { Índice de } \\
\text { Sobre } \\
\text { Esfuerzos } \\
\text {-Pañete } \\
\text { Reforzado } \\
\text { con Malla } \\
\text { de Vena }\end{array}$ & 1.17 & 0.99 & 0.82 & 0.82 & 0.64 & 0.9 \\
\hline $\begin{array}{l}\text { Pañete } \\
\text { reforzado } \\
\text { Permisible } \\
\text { Malla } \\
\text { Electro } \\
\text { soldada }\end{array}$ & 5.81 & 5.81 & 11.62 & 11.62 & 59 & 59 \\
\hline $\begin{array}{l}\text { Índice de } \\
\text { Sobre } \\
\text { Esfuerzos } \\
\text {-Pañete } \\
\text { Reforzado } \\
\text { con Malla } \\
\text { electro } \\
\text { soldada } \\
\end{array}$ & 0.86 & 0.72 & 0.6 & 0.6 & 0.64 & 0.9 \\
\hline
\end{tabular}

\section{E. Métodos de rehabilitación y consolidación}

Con base en los resultados de esta investigación, los avances metodológicos y analíticos desarrollados por la Asociación de Ingeniería Sísmica, AIS [15], a la luz de los lineamientos de la NSR-10 y la Ley 1185 del Ministerio de Cultura de Colombia, se plantean varios métodos de rehabilitación con base en cinco métodos primarios que, a su vez, combinados, ofrecen alternativas de consolidación; por ejemplo: pañete reforzado con malla de vena, pañete reforzado con malla electro soldada, estructura matriz de madera (incluye un retícula de madera chanul en las dos direcciones y dos caras de la tapia) y la estructura metálica en exoesqueleto, proyectada como un sistema arriostrado externo para estabilizar la zona lateral derecha (Fig. 26).

\section{a. Método uno: pañete reforzado con malla de vena PRMV}

Descripción: se aplica un pañete reforzado con malla de vena calibre 19 en las dos caras de la tapia y de manera simultánea, con una pasta de suelo cemento, a 1:10 (caracterizado por esta investigación), con fibra sintética (polipropileno) al $0.5 \%$ en peso del cemento.

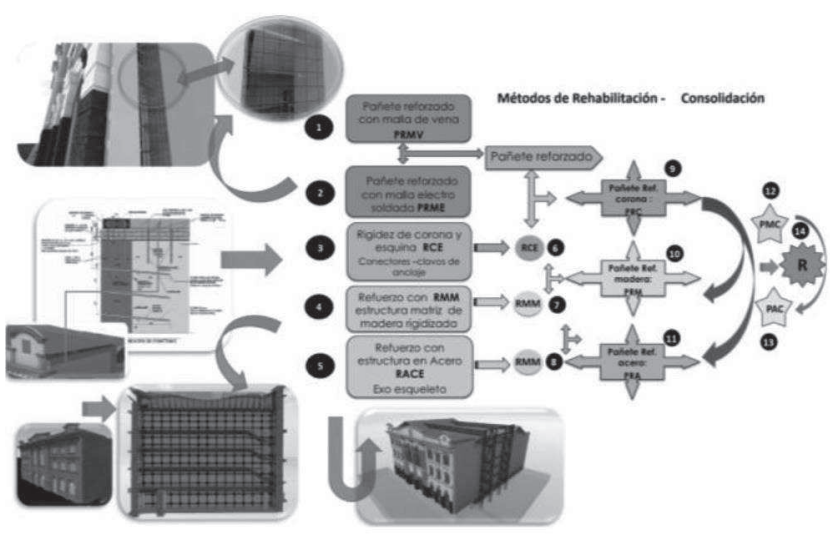

Fig. 26. Métodos de rehabilitación para el teatro imperial, opciones de consolidación del sistema murario en tapia.

\section{b. Método dos: pañete reforzado con malla electro soldada PRME}

Descripción: se aplica un pañete reforzado con malla electro soldada de $4 \mathrm{~mm}$ referencia comercial M084 Ø4 mm $15 \times 15$, en las dos caras de la tapia y de manera simultánea con una pasta de suelo cemento a (1:10 caracterizado por esta investigación) con fibra sintética (polipropileno) al $0.5 \%$ en peso del cemento.

\section{c. Método tres: refuerzo con estructura matriz de madera rigidizada con diagonales en varillas de acero RMM}

Descripción: consiste en el refuerzo de la tapia mediante el uso de una estructura matriz de madera $(0.20 \times 0.07 \mathrm{~m})$ en forma de retícula, separada en promedio $1.50 \mathrm{~m}$, en ambas caras de la tapia conectadas con un perno de $1 / 2$ " en cada nudo y este, a su vez, sobre una platina de $1 / 8$ " en los nudos y en cada lado. La estructura de madera va rigidizada con diagonales en varillas de acero en los extremos verticales y en las franjas superior, media e inferior.

\section{d. Método cuatro: refuerzo con estructura en Acero RACE sistema Exoesqueleto RACE}

Descripción: debido a la alta vulnerabilidad de la tapia lateral derecha, para estabilizarla, se proyecta como una cuarta alternativa un sistema aporticado y arriostrado con diagonales concéntricas en " $K$ invertida, en perfiles metálicos", cimentado sobre caisson, caracterizado por columnas HE300A, vigas HE240A, con diagonales HE260A. A su vez, el pórtico se conecta con las columnas internas existentes en acero, tipo tubo rectangular de 400x500x40mm, ubicadas en la boca del escenario a través de una cercha en tubo estructural con especificaciones de cuerda superior e inferior, diámetro $273.1 \mathrm{mmx} 12 \mathrm{~mm}$, las diagonales y montantes $152.4 \mathrm{mmx} 8 \mathrm{~mm}$ con uniones soldadas. Las conexiones entre viga y columna se realizan con conexiones end plate.

\section{Conclusiones}

Los parámetros estáticos promedio de la tapia en cuanto a la resistencia promedio a la compresión es de $0.59 \mathrm{Mpa}$., el 
módulo de Young es de 72.5Mpa, la relación de Poisson es 0.33 y el Módulo de esfuerzo cortante es 27.7 Mpa.

Los parámetros dinámicos promedio de la tapia en cuanto a velocidad primaria o de compresión es de $723 \mathrm{~m} / \mathrm{sg}$. Velocidad secundaria o de corte es de $304 \mathrm{~m} / \mathrm{sg}$, la relación de Poisson dinámica es 0.33

La vulnerabilidad sísmica de las edificaciones en tierra puede determinarse con base en el apoyo de ensayos estandarizados y adaptados a las condiciones de las características físico- mecánicas de la tapia, como un suelo consolidado con propiedades estáticas y dinámicas, que permiten un análisis estructural (no lineal y una exploración lineal).

Es importante destacar que los suelos que constituye la tapia son característicos, con predominancia granulares finos; tal es el caso de teatro Imperial, un limo de baja plasticidad. Así, con base en los límites, el grado de consistencia de la tapia es media dura sólida.

Además, la fábrica de tapia con métodos artesanales y aditivos orgánicos consigue materiales de suelos consolidados de alta resistencia que superan las $0.50 \mathrm{Mpa}$. La relación de las ondas de compresión respecto a las de corte en la tapia es de 2.4 veces su magnitud.

Con igual geometría y carga, la capacidad de respuesta al utilizar pañete reforzado con suelo cemento (10\%), fibra de polipropileno $(0.55 \%)$ con malla de vena mejora 4.9 veces y 10 veces con malla electro soldada. El comportamiento elástico en cuanto a deformación en la tapia reforzada con pañete, mejora 7 veces con malla de vena y 3 veces con malla electro soldada.

En comparación con la malla de vena, al conjugar las curvas de los especímenes con pañete reforzado, las de malla electro soldada mejoran un $20 \%$ la capacidad de respuesta. Asimismo, el comportamiento de las probetas consolidadas con pañete reforzado con malla electro soldada, ofrece menor deformación con cargas mayores. Las probetas consolidadas con pañete reforzado y malla electro soldada se comportan más rígidas. Por su parte, las probetas consolidadas con pañete reforzado con malla de vena, comparadas con aquellas electro soldadas, son más dúctiles y ofrecen mejores esfuerzos residuales.

El comportamiento dinámico de la tapia $\mathrm{y}$, en general, de las estructuras históricas, no alcanzan a disipar esfuerzos y ofrecer una respuesta a un período de retorno de 45 años con un ciclo corto. Para el caso de teatro Imperial, hipotéticamente existe probabilidad de colapso para una relación media de daño $\mathrm{RMD}=1$.

\section{AgradeCIMIENTOS}

Universidad de Nariño, Universidad de Valle, Arq. Ph.D. William Pasuy A., Arq. Ana Matilde Vicuña, Arq. Martha Enríquez, Ing. Esp. Carlos Rosero e Ing. Ph.D. Manolo Galván. Ingeniero M.Sc. Carlos Bucheli, Ing. Javier Solarte, Ing. Héctor Collazos y Jair Revelo.

\section{REFERENCIAS}

[1] Cely, F, \& Y., Patrimonio y arquitectura en tierra. Avances de investigación. Bogotá: Pontificia Universidad Javeriana, 2009.
[2] Enríquez, G., Mesías, R., y Ortega, E., Pasto Republicano. San Juan de Pasto, Imprelibros. S.A, 2005.

[3] Hugo, C., Reseña Histórica de los Terremotos en Nariño. Pasto, 2004.

[4] W, P., Hernández, R. y Olimpia, N., Experiencias y Métodos de Restauración en Colombia, Teatro Imperial de Pasto un Patrimonio Vivo. Roma, Aracne editrice Roma, 2011.

[5] Morán, M., Crespo A. y Ormaza. Reforzamiento estructural en las edificaciones patrimoniales. Quito: Fonsal, 2004.

[6] Sísmica, A. C., Reglamento colombiano de construcción sismo resistente. NSR - 10. Santafé de Bogotá, 2012.

[7] ISCARSAH, Recomendaciones para el análisis, conservación y restauración estructural del patrimonio arquitectónico. Barcelona, Icomos, 2004.

[8] Moretti, V., Doglioni, A. y Petrini, F., Churches and Earquakes. Milán, Triest, 1994

[9] Tovar, \& D., Determinación de los módulos dinámicos para un suelo lacustre de Bogotá mediante ensayo triaxial cíclico. Bogotá D.C., Universidad de la Salle, 2016.

[10] Reyes, \& P., Ensayos para la obtención de propiedades dinámicas de un suelo mh estabilizado con caucho triturado, Cali, Universidad del Valle, 2017.

[11] Giancarlo, \& D., Surface wave analysis for near surface aplications Elsevier USA, Elsevier First Edit, 2015.

[12] Título, Grupo Geotécnica, Universidad Cantabria, 29 de junio de 2017, junio. www.google.com. Recuperado de: http://ocw.unican.es/ ensenanzas-tecnicas/geotecnia-i/materiales-de-clase/capitulo6.pdf

[13] Paulay, T, \& M., Seismic Design Of Reinforced Concrete And Masonry Buildings. United States: John Wiley \& Sons, Inc., 1992.

[14] Bonnet, Serna, \& Dorado y Rosero., Consultoría para la descripción evaluación estructural y de servicio en edificaciones indispensables y estructuras de ocupación especial en Pasto de acuerdo al riesgo sísmico. Pasto, 2015.

[15] Sísmica, A. C., Manual para la rehabilitación de viviendas construidas en adobe y tapia pisada. Bogotá, 2004.

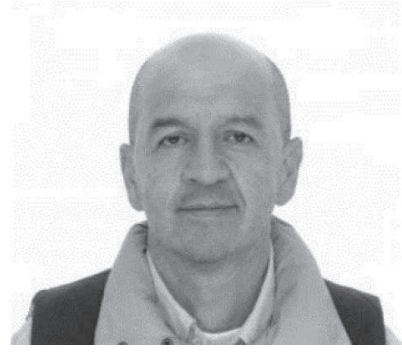

William Castillo Valencia. Nació en Pasto, Nariño. Se graduó como Ingeniero Civil en la Universidad de Nariño y obtuvo su título como especialista en estructuras; se encuentra postulado como maestrante en Ingeniería, énfasis en Ingeniería Civil con orientación estructuras de la Universidad del Valle; pertenece al grupo de investigación G7 en Ingeniería Sísmica Eólica y Estructuras. Ha sido ponente internacional en países como Cuba, México, Guatemala, Perú, EE.UU. y Argentina. Actualmente es profesor de tiempo completo de la Universidad de Nariño, profesor internacional invitado de la Universidad Michoacana San Nicolás de Hidalgo- Morelia, México. Es investigador del observatorio de culturas urbanas, OCUR, de la Universidad de Nariño. Ha recibido premios de reconocimiento por representaciones internacionales de la rectoría de la Universidad de Nariño y se desempeña como consultor en estructuras; miembro activo de la Asociación Colombiana de Ingeniería Sísmica.

ID de registro ORCID 0000-0002-5739-6494

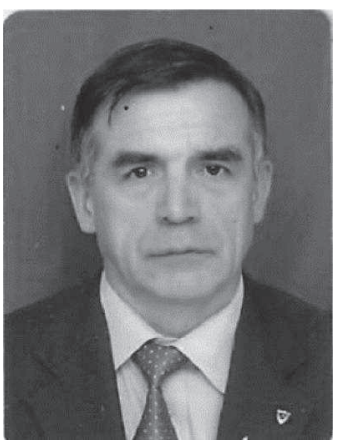

Hugo Coral Moncayo. Nació en San Bernardo, Nariño. Ingeniero Civil de la Universidad del Cauca, doctor en Ingeniería Sísmica y Dinámica Estructural de la Universidad Politécnica de Cataluña, magister en Geotecnia de la Universidad Nacional de Colombia, profesor del postgrado de la maestría en Ingeniería con énfasis en Ingeniería Civil orientación Geotecnia. Recibió mención de felicitaciones en reconocimiento a la labor académica realizada en Acofi y Ecaes; consultor en geotecnia de la firma 
Ingeniería de Suelos y Cimentaciones; se desempeñó como profesor tiempo completo de la Universidad de Nariño en pregrado y postgrados, investigador del grupo G7 de la Universidad del Valle, miembro activo de la Asociación Colombiana de Ingeniería Sísmica.

ID de registro ORCID 0000-0002-7019-9479

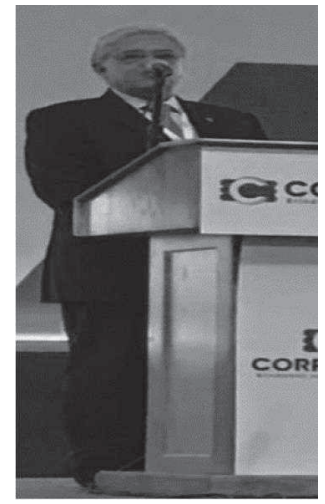

Gilberto Areiza Palma. Ingeniero Civil de la Universidad del Valle, master en Ingeniería Estructural de la Universidad de Lehigh, EU, profesor de tiempo completo de pregrado y postgrado de la maestría en Ingeniería con énfasis en Ingeniería Civil orientación estructuras. Profesor de la especialización en Estructuras de la Universidad del Valle, área de actuación ingeniería y tecnología. Es miembro del grupo de investigación G7 de la Universidad del Valle, dinámica eólica y estructuras; miembro fundador del Instituto Colombiano del Acero, autor de publicaciones bibliográficas, seminarios y conferencias; investigador del desempeño sísmico de edificaciones de

muros estructurales delgados en concreto reforzado. Consultor de la firma consultora GAP. INGENIERÍA SAS, miembro activo de la Asociación Colombiana de Ingeniería Sísmica e Instituto Colombiano del Acero.

ID de registro ORCID 0000-0002-8028-5573 